doi:10.1017/\$1041610221002775

\section{A retrospective analysis of patient flow in mental health services for older adults in South London during the COVID-19 pandemic}

While older mental health patients had high mortality (Perera et al., 2021) during the first wave of the COVID-19 pandemic, referrals to mental health services for older people decreased substantially in the UK (Greig et al., 2021). This study aimed to investigate patient flow in and out of community mental health services for older adults during the first and second wave of the COVID-19 pandemic and the period in-between.

We carried out a retrospective analysis of mental health services' caseload, referrals, and discharges from March 2019 to February 2021 using data from the Clinical Record Interactive Search (CRIS) system. CRIS provides researchers access to more than 500,000 anonymised mental health and dementia care records for patients under the South London and Maudsley NHS Foundation Trust (SLaM) and has full approval for secondary analysis by the Oxford Research Ethics Committee C (reference: 18/SC/0372).

We defined three "pandemic" 4-month time periods [first wave (March-June 2020), intermediate period (July-October 2020), and second wave (November 2020-February 2021)] and three corresponding pre-pandemic periods. Patient flow data were ascertained from community mental health services for older adults, which in SLaM comprise Community Mental Health Teams, Care Home Intervention Teams and Memory Services. For the six time periods, we calculated mean daily caseloads, as well as mean weekly accepted referrals and discharges for all community services combined. We carried out independent unpaired t-test to determine if there is a statistically significant difference between two periods.

The mean daily caseload ( $95 \%$ confidence interval (CI)) for the pre-pandemic period (March 2019February 2020) was 2291 (2286-2300) and was lower during pandemic period (March 2020-February 2021) with 1970 (1960-1980; p-value <0.001). In the pre-pandemic period, the mean $(95 \% \mathrm{CI})$ weekly number of accepted referrals was 89 (85-93) and discharges was 87 (83-91), while in the pandemic period both were significantly lower ( $p$-value $<0.001$ ) with 65 (60-70) accepted referrals and 72 (66-78) discharges per week.
Figure 1 shows the mean daily caseload (1a), mean weekly accepted referrals (1b), and mean weekly discharges (1c) with $95 \%$ CI for the six 4-month time periods. The mean daily caseload (Figure 1a) was significantly lower during the first wave (March-June 2020) than in any of the prepandemic time periods. During the pandemic, no further statistically significant decline in daily caseload occurred. There was a sharp decline in accepted referrals (Figure 1b) during the first wave compared to any of the pre-pandemic periods, but during JulyOctober 2020 the number of accepted referrals increased again. We detected a significantly higher number of accepted referrals during the second compared to the first wave. Interestingly, there was no difference in the mean weekly discharges during the first wave compared to any pre-pandemic period, and no difference across the pandemic time periods. However, fewer patients were discharged in JulyOctober 2020 and during the second wave compared to the respective pre-pandemic episodes.

As previously described (Greig et al., 2021), we saw a drop in cases under specialist mental health services for older people during the first wave of the COVID-19 pandemic. While referrals and caseload did not recover to pre-pandemic levels during the summer of 2020, no further decline was noted during the second wave. This indicates that by the time of the second wave of the COVID-19 pandemic, mental health services for older people had adapted to working under pandemic conditions, with for example more protective equipment and access to remote consultations (Mok et al., 2020). The reduction in caseload appears largely driven by fewer accepted referrals. The number of discharges from secondary care remained unchanged or fell during the pandemic period, indicating that mental health services continued to support patients already under their care. Those with new onset mental health problems, however, might have been missed by services. The reasons for this could be fewer referrals by GPs/primary care, and indeed the number of mental health presentations recorded in primary care data during the early months of the pandemic was lower (Carr et al., 2021), but also through avoidance of consultations by patients/families due to restrictions or fear of getting COVID-19, or lack of access to technology for virtual assessments (Banerjee, 2020).

In conclusion, the caseload of older people's mental health services remained stable when the second wave of the COVID-19 pandemic hit the UK. This shows that old age psychiatry services 

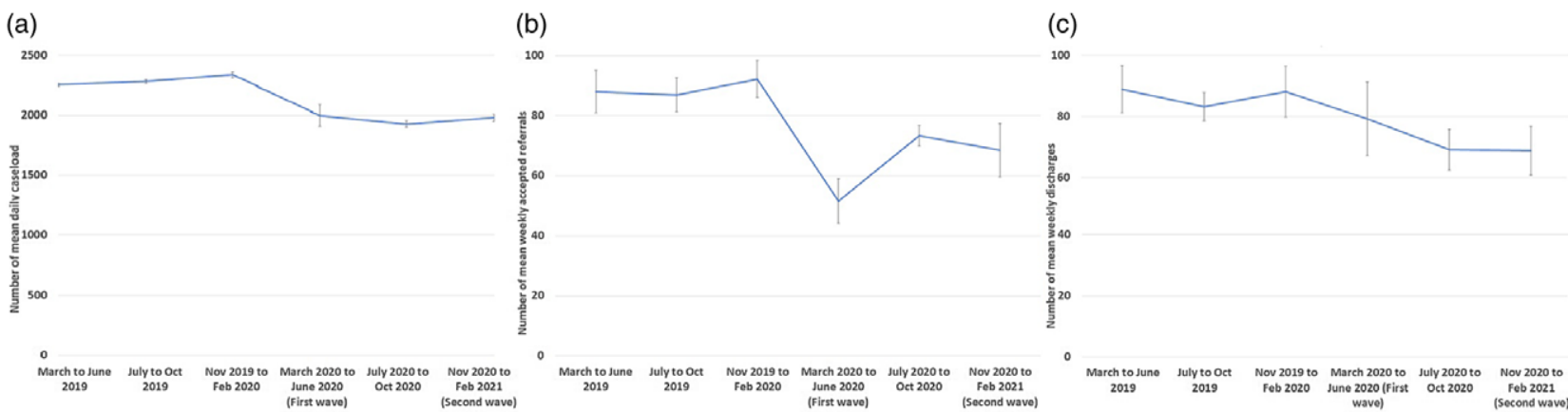

Figure 1. Old age psychiatry service use before and during the COVID-19 pandemic. (a) Mean daily caseload ( $95 \%$ confidence interval). (b) Mean weekly accepted referrals ( $95 \%$ confidence interval). (c) Mean weekly discharges ( $95 \%$ confidence interval).

adapted to working under pandemic circumstances. More proactive preparation and earlier adaptation of new ways of working appears to be key for future pandemics, whereby ensuring save and lowthreshold access to mental health care is crucial.

\section{Conflict of interest}

RS has received recent research funding from Roche, Janssen, GSK, and Takeda. GP, CM, $\mathrm{MB}$, and LV declare no conflict of interest.

\section{Source of funding}

The data resource, GP, CM, and RS are partfunded by the National Institute for Health Research (NIHR) Biomedical Research Centre at South London and Maudsley NHS Foundation Trust and King's College London, and RS by the National Institute for Health Research (NIHR) Applied Research Collaboration South London (NIHR ARC South London) at King's College Hospital NHS Foundation Trust. The views expressed are those of the authors and not necessarily those of the NHS, the NIHR or the Department of Health.

\section{Description of authors' roles}

The study was conceived by LV and CM. Analyses were carried out by GP and CM. The manuscript was written by CM and finalized by LV and with substantial text contribution from all authors.

\section{Data sharing statement}

No additional data are available.

\section{References}

Banerjee, D. (2020). The impact of Covid-19 pandemic on elderly mental health. International fournal of Geriatric Psychiatry, 35, 1466-1467.

Carr, M. J. et al. (2021). Effects of the COVID-19 pandemic on primary care-recorded mental illness and self-harm episodes in the UK: a population-based cohort study. The Lancet Public Health, 6, e124-e135.

Greig, F., Perera, G., Tsamakis, K., Stewart, R., Velayudhan, L. and Mueller, C. (2021). Loneliness in older adult mental health services during the COVID-19 pandemic and before: associations with disability, functioning and pharmacotherapy. International fournal of Geriatric Psychiatry, 40, 218.

Mok, V. C. T. et al. (2020). Tackling challenges in care of Alzheimer's disease and other dementias amid the COVID-19 pandemic, now and in the future. Alzheimer's Dementia, 16, 1571-1581.

Perera, G., Mueller, C., Broadbent, M., Stewart, R. and Velayudhan, L. (2021). Mortality among mental health services for older adults during the COVID-19 pandemic: a retrospective analysis from South London. International Psychogeriatrics, 33, 527-528.

Christoph Mueller, ${ }^{1,2 \dagger}{ }^{2}$ G Gayan Perera, ${ }^{1 \dagger}$ Matthew Broadbent, ${ }^{2}$ Robert Stewart ${ }^{1,2}$ AND Latha Velayudhan ${ }^{1,2}$

${ }^{1}$ Institute of Psychiatry, Psychology and Neuroscience, King's College London, London, UK

${ }^{2}$ South London and Maudsley NHS Foundation Trust, London, UK

Correspondence should be addressed to: Christoph Mueller, MD, Institute of Psychiatry, Psychology and Neuroscience (IoPPN), King's College London, De Crespigny Park, London, SE5 8AF, UK. Tel: +44207848 0626. Email: christoph.mueller@kcl.ac.uk

† Christoph Mueller and Gayan Perera are shared co-first authors who have worked together and contributed equally. 